\title{
Seizure control after subtotal lesional resection
}

\author{
William C. Gump, M.D., ${ }^{1,3}$ Karen L. SkJei, M.D., ${ }^{2,3}$ and Shefali N. Karkare, M.D. ${ }^{4}$ \\ ${ }^{1}$ Norton Neuroscience Institute Division of Pediatric Neurosurgery; ${ }^{2}$ University of Louisville Division of \\ Child Neurology; ${ }^{3}$ Kosair Children's Hospital, Louisville, Kentucky; and ${ }^{4}$ Steven and Alexandra Cohen \\ Children's Medical Center, New Hyde Park, New York
}

\begin{abstract}
Reports on seizure outcomes following surgery for lesional epilepsy consistently cite extent of resection as a significant predictor of outcome. Unfortunately, gross-total resection is not technically feasible in all cases of medically refractory tumor-associated epilepsy. Here, the authors present the case of a 4-year-old girl whose epilepsy was medically controlled after 1-stage electrocorticography-guided subtotal resection (STR) of a large diffuse protoplasmic astrocytoma. They also review the modern literature on epilepsy associated with brain tumors. Outcomes are compared with those following surgical treatment of focal cortical dysplasia and vascular lesions. Gross-total lesional resection shows significant superiority across pathologies and anatomical regions. Despite a considerable number of STRs yielding seizure freedom, other favorable treatment factors have not been defined. Although gross-total lesional resection, if possible, is clearly superior, tailored surgery may still offer patients a significant opportunity for a good outcome. Treatment factors yielding successful seizure control following STR remain to be fully elucidated. (http://thejns.org/doi/abs/10.3171/2013.3.FOCUS1348)
\end{abstract}

KEY WoRDS $\bullet \quad$ epilepsy $\bullet \quad$ subtotal resection $\bullet \quad$ seizure control •
gross-total resection

$\mathrm{I}$ NTRACRANIAL mass lesions such as brain tumors have a well-established association with seizures. ${ }^{33}$ Lesion type and location are both relevant. Overall, 20\%$40 \%$ of patients with brain tumors experience at least 1 seizure prior to diagnosis, and another $20 \%-45 \%$ will experience seizures at some point following diagnosis. ${ }^{24,29,31}$ Low-grade gliomas have been relatively more epileptogenic than malignant tumors. ${ }^{13}$ In patients harboring both brain tumors and refractory epilepsy, the challenge is to optimize therapy for both disease processes, which can evolve independently or in tandem.

Although GTR consistently appears to be a dominant factor for a good postoperative seizure outcome, some brain tumors may not be resectable, particularly in the case of large, infiltrative low-grade lesions. The role of chemotherapy and radiation for seizure control is not well defined in this context. ${ }^{32}$ Surgical strategies for optimizing epilepsy outcomes must be carefully considered in cases such as these.

\footnotetext{
Abbreviations used in this paper: DNET $=$ dysembryoplastic neuroepithelial tumor; ECoG = electrocorticography; GTR = grosstotal resection; ILAE = International League Against Epilepsy; STR $=$ subtotal resection.
}

\section{Illustrative Case}

History and Examination. A 4-year-old girl presented to our Comprehensive Epilepsy Center in Louisville having experienced about 3 months of generalized seizures, which were increasing in frequency. Neurological examination was nonfocal. Magnetic resonance imaging of the brain revealed an extensive diffuse nonenhancing area of abnormal signal involving the right frontal and temporal cortex and subcortical structures (Fig. 1). There was minimal mass effect and no restricted diffusion.

The patient was taken to surgery for a biopsy, which yielded an initial diagnosis of nonspecific low-grade glioma. Medical control of the patient's seizures gradually deteriorated despite polypharmacy and no radiographic tumor progression. She did not tolerate topiramate. Seizures localized primarily to the right temporal lobe, as assessed by seizure semiology, prolonged videoelectroencephalography monitoring, and PET scanning. Limbic structures on the right side did not appear sclerotic or invaded by tumor on MRI but were relatively hypometabolic on PET scanning (Fig. 2).

Operation. Eight months after the initial biopsy, the patient returned to surgery for an anterior temporal lo- 

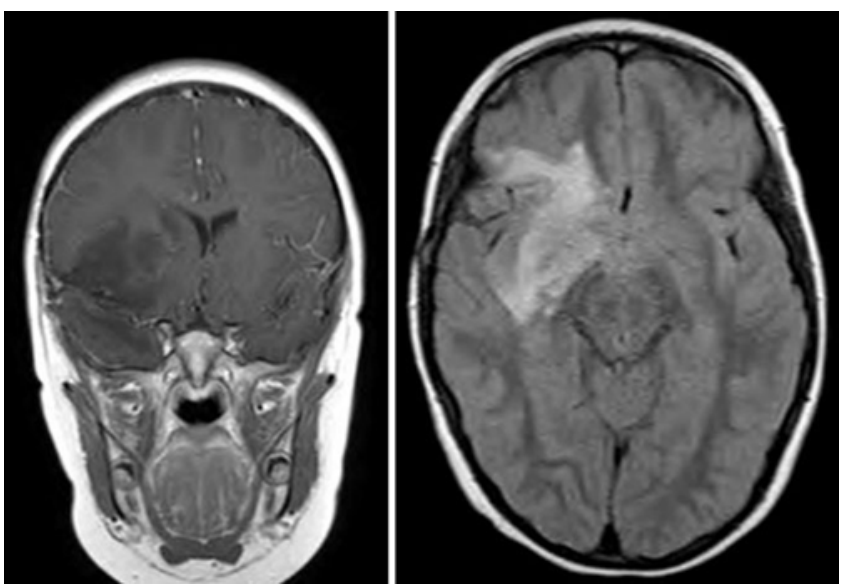

FIG. 1. Coronal T1-weighted MR image with contrast and axial FLAIR MR image showing a diffuse nonenhancing area of signal abnormality involving the cortex and subcortical structures in the right frontal and temporal lobes.

bectomy and amygdalohippocampectomy (Fig. 3). Intraoperative ECoG, used to localize areas of interictal spiking, guided further temporal and frontal cortical resection. Based on an additional tissue specimen from this procedure, the ultimate diagnosis of protoplasmic astrocytoma (WHO Grade II) was made.

Postoperative Course. The patient remained neurologically intact postoperatively. Seizure frequency decreased over the following 3 months with additional changes in medications and dosing. She has been seizure free on a regimen of clobazam, lacosamide, levetiracetam, and oxcarbazepine. No further adjuvant tumor therapy was initiated over this time.

\section{Discussion}

No study has demonstrated seizure outcomes similar or superior to those following subtotal tumor resection, when compared directly with GTR, except in 1 series of high-grade gliomas. ${ }^{7}$ This includes case series in which ECoG was used to localize interictal spikes intraoperatively and ictal onset extraoperatively.8,20,23,28 Although GTR is not always technically achievable, this limitation does not necessarily leave patients in these cases with ongoing uncontrolled epilepsy. However, the treatment factors that could predispose toward a good outcome in the context of STR have not been well established.
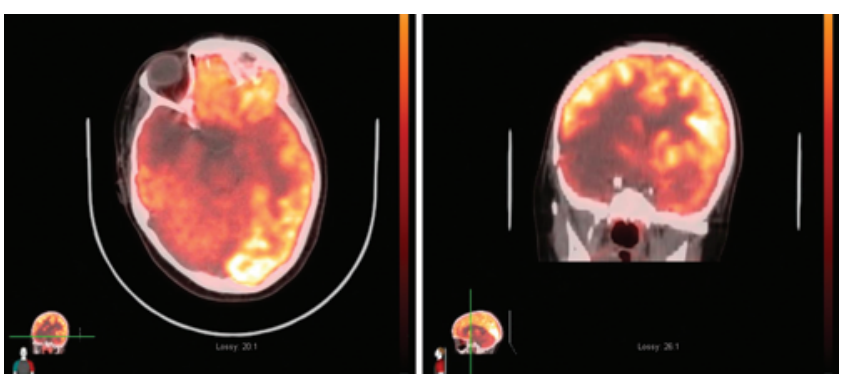

FIG. 2. Preoperative axial and coronal PET scans showing hypometabolism in the right temporal lobe, particularly within the mesial structures.
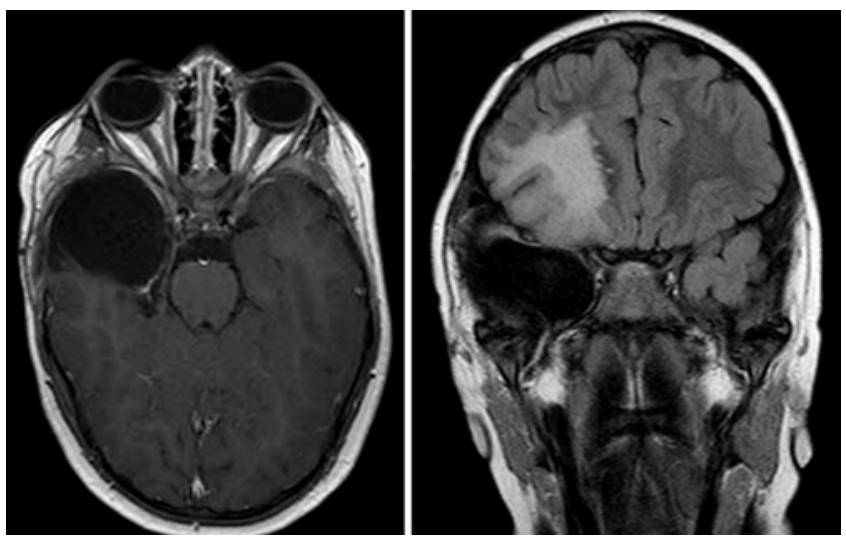

FIG. 3. Postoperative axial T1-weighted MR image with contrast and coronal FLAIR MR image demonstrating anterior temporal lobectomy with amygdalohippocampectomy, with significant residual tumor in the frontal lobe.

Authors of numerous retrospective case studies have reviewed seizure outcomes after brain tumor surgery from both pathological and anatomical perspectives. Representative results are summarized in Table 1. Many large studies of low-grade gliomas have been accumulated; $5,6,9,26,37,38$ only 1 has specifically focused on high-grade glioma. ${ }^{7}$ Glioneuronal tumors, specifically ganglioglioma and DNET, are often considered together ${ }^{3,18}$ because of their overlapping clinical and pathological characteristics, including epileptogenicity. Seizure outcomes have also been specifically examined for temporal lobe ${ }^{14}$ and insular cortex ${ }^{10,36}$ tumors. Seizure outcomes for temporal lobe and insular cortex tumors are comparable to those achieved after surgery for nonneoplastic entities including vascular pathologies, such as cavernous malformation $^{15}$ and arteriovenous malformation, ${ }^{17,19}$ and focal cortical dysplasia. ${ }^{30}$

\section{Oncological Perspective}

Low-grade gliomas are the most common neoplastic pathology associated with pharmacoresistant epilepsy. ${ }^{28,32}$ A recent review ${ }^{12}$ of reports published between 1985 and 2010 revealed 773 patients with low-grade gliomas across 20 studies in which seizure outcomes after 6 months or more postoperatively were recorded. Gross-total resection was most predictive of complete seizure freedom, which was achieved in $357(80 \%)$ of 448 patients who had undergone GTR. Only 100 (53\%) of 187 patients who had undergone STR achieved an Engel Class I outcome.

TABLE 1: Postoperative seizure outcomes from selected meta-analyses

\begin{tabular}{llc}
\hline \multicolumn{1}{c}{ Authors \& Year } & \multicolumn{1}{c}{ Pathology } & $\begin{array}{c}\text { Engel Class I } \\
\text { Outcomes (\%) }\end{array}$ \\
\hline Englot et al., 2012 & low-grade glioma* & 53 \\
Englot et al., 2011 & ganglioglioma/DNET* & 55 \\
Rowland et al., 2012 & focal cortical dysplasia & 55.8 \\
Vale et al., 2012 & mesial temporal sclerosis & 78.9 \\
\hline
\end{tabular}

* Treated with STR. 


\section{Subtotal resection}

Other predictors of seizure freedom included good preoperative medical control of epilepsy, epilepsy duration < 1 year, and seizure semiology other than simple partial. There was no difference in seizure outcome between temporal lobe and extratemporal tumors. The use of intraoperative ECoG did not improve outcome, although its use was inconsistent within and across the various studies.

A subsequent large, single-institution retrospective analysis $^{37}$ of 183 patients with low-grade gliomas showed consistent results, with 43 (78\%) of 55 GTRs yielding an Engel Class I outcome, as compared with 52 (49\%) of 105 STRs. Loss of heterozygosity on chromosome $19 \mathrm{q}$ and a lower Ki-67 index were pathological findings predicting better seizure outcomes in this study. The use of ECoG during surgery was based on surgeon preference. No additional treatment-related predictors of seizure freedom were identified.

Only 1 study $^{7}$ has systematically examined seizure outcomes in patients with malignant glioma (WHO Grade III or IV), even though this lesion is the most common primary brain tumor in adults. Note, however, that only 153 (24\%) of 648 patients initially presented with seizures, a much lower rate than has been observed in lower-grade pathologies. Seizures were more common in patients harboring anaplastic astrocytoma than glioblastoma multiforme. In this study, the extent of resection was not significantly associated with postoperative seizure freedom, although seizure recurrence after postoperative seizure freedom was strongly correlated with tumor recurrence. A higher Karnofsky Performance Scale score, a tumor location outside the parietal lobe, and the absence of preoperative uncontrolled seizures were factors that corresponded to a better postoperative seizure outcome in patients with preoperative seizures. Long-term outcome analyses in this population are limited by significantly worse overall survival compared with those for other pathologies.

A 2012 review of 39 studies on seizure outcome following resection of ganglioglioma or $\mathrm{DNET}^{11}$ documented an overall Engel Class I outcome post-GTR in 552 (87\%) of 632 patients with 1 of these pathologies. The superiority of these results, as compared to an Engel Class I outcome post-STR in 88 (55\%) of 160 patients, reached statistical significance. Other statistically significant factors included duration of epilepsy $(<1$ year was associated with a better outcome) and seizure semiology. The preoperative presence of secondarily generalized seizures predicted a lower rate of seizure freedom than that in patients with partial seizures only. Outcomes were not significantly different when comparing a temporal versus an extratemporal tumor location. Neither did the use of intraoperative ECoG demonstrate a statistically significant effect in this analysis, although again its utilization was not standardized across the pooled studies.

A subsequent report ${ }^{20}$ describing 55 patients with epileptogenic ganglioglioma revealed 48 (87\%) Engel Class I outcomes, consistent with prior studies, but did not show extent of resection to be a significant predictor of seizure outcome. Intraoperative ECoG, used in 42 patients, also did not affect outcome, and neither did a temporal versus an extratemporal tumor location. Another recent report, which was also limited to ganglioglioma pathology, ${ }^{34}$ demonstrated more seizures and more tumor progression with STR; ECoG was used in some patients according to individual surgeon preference. As in the low-grade glioma meta-analysis,${ }^{12}$ duration of epilepsy $<1$ year was associated with a better seizure outcome.

A more recent retrospective case study of pediatric patients with low-grade brain tumor diagnosis and associated seizures ${ }^{5}$ compared lesionectomy outcomes with the results of tailored resections, with a minimum follow-up of 1 year. Pathological diagnoses included mostly ganglioglioma and DNET, sometimes with associated focal cortical dysplasia identified. The tailored resection arm included only patients with temporal lobe tumors, in whom an extensive noninvasive preoperative epilepsy evaluation was performed. There were 5 right-sided and 6 left-sided tumors. A standard anterior temporal lobectomy with resection of mesial structures was performed and encompassed the tumor entirely. The use of ECoG was not mentioned. In the lesionectomy group, 17 of 20 patients underwent GTR; among these patients were 14 Engel Class I, 2 Engel Class II, and 1 Engel Class III outcomes. Two patients who underwent STR also achieved an Engel Class I outcome, and a third patient had an Engel Class II outcome. All 11 patients in the tailored resection group achieved Engel Class I outcomes.

\section{Anatomical Perspective}

Temporal lobe tumors have received specific attention, as mesial temporal structures are known to be associated with intractable epilepsy and are accessible within the surgical field. A recent systematic literature review of seizure outcomes following temporal lobe surgery for low-grade tumors ${ }^{14}$ demonstrated the best results when extended resection with corticectomy and hippocampectomy were performed. The study population all harbored WHO Grade I or II pathology, and patients were followed up for a minimum of 6 months postoperatively. Eighty-seven percent (294 of 338) of the patients had an Engel Class I outcome after surgery. This result was very similar to the $86.8 \%$ Engel Class I outcomes in patients who underwent GTR plus extended cortical resection and the $86 \%$ Engel Class I outcomes in patients who underwent GTR plus hippocampectomy. However, 78.6\% (327 of 416) of the patients who underwent GTR alone also had Engel Class I outcomes. The difference with STR patients was highly significant; only $42.7 \%$ (70 of 164) of patients in this group had Engel Class I outcomes. The use of intraoperative and/ or extraoperative ECoG or other adjuncts in the STR group was not standardized across the various studies pooled.

Two recent small case studies ${ }^{10,36}$ on insular tumors showed very good overall results for seizure control. One report on 11 patients, ${ }^{10}$ all with diagnosed low-grade glioma, demonstrated an Engel Class I outcome after 3 of 3 total resections and 6 of 8 STRs. Another study ${ }^{36}$ of 24 patients included 13 WHO Grade I-III tumors and 11 nonneoplastic lesions. Seizure outcomes were reported according to the ILAE scale; 13 of 17 GTR cases had ILAE 1 or 2 outcomes, compared with 4 of 7 STR cases. Both studies suggested that good results are attainable with these technically very challenging lesions and that extent of resection also predicts a better outcome. 


\section{Nononcological Perspective}

Comparison with other primary seizure disorders can help better contextualize tumor resection outcomes. Mesial temporal sclerosis is the most common preoperative diagnosis in adults undergoing epilepsy surgery, and its results stand as a de facto gold standard for assessing surgical seizure outcomes. A recent review ${ }^{35}$ of large modern studies revealed overall Engel Class I outcomes in 541 (78.9\%) of 686 patients who had undergone surgery for this condition. Resection is, in general, somewhat more successful than other epilepsy operations, such as hemispherectomy ${ }^{25}$ for Rasmussen encephalitis (about 70\% Engel Class I), SturgeWeber syndrome (same), or hemimegalencephaly (approximately 50\% Engel Class I). Palliative epilepsy interventions, such as vagus nerve stimulator placement or corpus callosotomy, are less likely to result in seizure freedom.

Seizure outcomes following subtotal tumor resection appear comparable to those after temporal lobe and extratemporal epilepsy operations in which the underlying etiology is focal cortical dysplasia. A recent metaanalysis encompassing 2014 patients with a pathological diagnosis of focal cortical dysplasia ${ }^{30}$ demonstrated an overall mean postoperative seizure freedom rate of $55.8 \%$ $\pm 16.2 \%$. Similar to findings after tumor resections, the most important treatment-related factor in focal cortical dysplasia was complete resection of the anatomical or electrographic abnormality. This result was reflected both in the collective data and in 14 of 37 individually reviewed studies. Other favorable factors included partial seizures, temporal lobe location, abnormality on MRI, and more abnormal histology.

Frontal lobe epilepsy surgeries have been similarly reviewed. ${ }^{16}$ Among 1199 patients in 21 studies, overall Engel Class I outcomes over 48 months or longer were found in only $45.1 \%$. The presence of an identifiable epileptogenic lesion preoperatively was a factor associated with a better postoperative seizure outcome. Among the 345 patients who had an identifiable epileptogenic lesion in the frontal lobe, GTR was strongly associated with a better outcome. One hundred thirty-eight (60.8\%) of 227 GTR cases were classified as having Engel Class I outcomes, compared with $43(36.4 \%)$ of 118 STR cases. As in the previously cited studies on tumors, this difference was highly statistically significant.

Nonlesional extratemporal epilepsy has been specifically reviewed in both adults ${ }^{2}$ and children. ${ }^{1}$ Of the 131 adults included in the meta-analysis, $45.8 \%$ had an Engel Class I outcome at 1 year postoperatively. Findings were somewhat less successful in the pediatric population, of whom only $33.7 \%$ (32 of 95 patients) achieved an Engel Class I result. No treatment-related factors in either study appeared to impact outcomes.

Seizures associated with cerebrovascular lesions have also been studied. A recent review of seizures associated with supratentorial cavernous malformations ${ }^{15}$ revealed gross-total lesional resection was strongly predictive of postoperative seizure freedom, although extended resection of the surrounding hemosiderin ring was not. A recent single-institution report on seizures after surgical treatment of supratentorial arteriovenous malformations ${ }^{17}$ showed excellent seizure results overall with $96 \%$ Engel
Class I outcomes, although complete lesional obliteration was not associated with better seizure outcomes in this series. This finding was unique among all studies reviewed, except the single high-grade glioma study. ${ }^{7}$ An earlier report, ${ }^{19}$ in contrast, did show that residual arteriovenous malformation was a seizure risk factor.

Nonsurgical modifiable treatment factors are relatively limited and have not been well defined at this time. A recent single-institution retrospective study ${ }^{22}$ of 312 patients indicated that those who underwent temporal lobectomy for intractable temporal lobe epilepsy seemed to have better postoperative seizure control when levetiracetam was part of their antiepileptic drug regimen. This finding was consistent with data in earlier, smaller studies $^{21,27}$ but contrary to results in a similar study (226 patients) ${ }^{4}$ that showed no relationship between postoperative seizure control and choice of antiepileptic drug. Although no conclusions have been reached at this time, the use of specific agents such as levetiracetam may be a potential new, modifiable seizure outcome predictor after surgery.

\section{Conclusions}

Surgical control of epilepsy is an appropriate treatment goal in the setting of a nonresectable lesion together with medically intractable seizures. Regarding the case featured in this paper, we hypothesize that medications and surgery may have worked synergistically to ultimately achieve full seizure control, although we discovered little definitive data that would support this view. Unfortunately, prospective data are not available to guide treatment decisions. Tumor location in the temporal lobe appears to be associated with a more favorable outcome, although no conclusions can be drawn at this time regarding the utility of extended cortical and/or mesial temporal resection in unresectable lesions. Similarly, the role of ECoG remains undetermined because of its inconsistent utilization across case studies. Strategies to optimize outcome in the context of STR have not been fully developed. The prospective study of seizure control efficacy using adjuncts such as intraoperative and extraoperative ECoG may offer the best opportunity to optimize surgical outcomes.

\section{Disclosure}

The authors report no conflict of interest concerning the materials or methods used in this study or the findings specified in this paper.

Author contributions to the study and manuscript preparation include the following. Conception and design: Gump. Acquisition of data: Gump. Analysis and interpretation of data: Gump. Drafting the article: all authors. Critically revising the article: all authors. Reviewed submitted version of manuscript: all authors. Approved the final version of the manuscript on behalf of all authors: Gump.

\section{References}

1. Ansari SF, Maher CO, Tubbs RS, Terry CL, Cohen-Gadol AA: Surgery for extratemporal nonlesional epilepsy in children: a meta-analysis. Childs Nerv Syst 26:945-951, 2010

2. Ansari SF, Tubbs RS, Terry CL, Cohen-Gadol AA: Surgery for extratemporal nonlesional epilepsy in adults: an outcome meta-analysis. Acta Neurochir (Wien) 152:1299-1305, 2010 
3. Aronica E, Leenstra S, van Veelen CWM, van Rijen PC, Hulsebos TJ, Tersmette AC, et al: Glioneuronal tumors and medically intractable epilepsy: a clinical study with long-term follow-up of seizure outcome after surgery. Epilepsy Res 43: 179-191, 2001

4. Asadi-Pooya AA, Nei M, Sharan AD, Mintzer S, Zangaladze A, Evans JG, et al: Antiepileptic drugs and relapse after epilepsy surgery. Epileptic Disord 10:193-198, 2008

5. Babini M, Giulioni M, Galassi E, Marucci G, Martinoni M, Rubboli G, et al: Seizure outcome of surgical treatment of focal epilepsy associated with low-grade tumors in children. Clinical article. J Neurosurg Pediatr 11:214-223, 2013

6. Benzagmout M, Gatignol P, Duffau H: Resection of World Health Organization Grade II gliomas involving Broca's area: methodological and functional considerations. Neurosurgery 61:741-753, 2007

7. Chaichana KL, Parker SL, Olivi A, Quiñones-Hinojosa A: Long-term seizure outcomes in adult patients undergoing primary resection of malignant brain astrocytomas. Clinical article. J Neurosurg 111:282-292, 2009

8. Chang EF, Christie C, Sullivan JE, Garcia PA, Tihan T, Gupta $\mathrm{N}$, et al: Seizure control outcomes after resection of dysembryoplastic neuroepithelial tumor in 50 patients. Clinical article. J Neurosurg Pediatr 5:123-130, 2010

9. Chang EF, Potts MB, Keles GE, Lamborn KR, Chang SM, Barbaro NM, et al: Seizure characteristics and control following resection in 332 patients with low-grade gliomas. J Neurosurg 108:227-235, 2008

10. Duffau H, Capelle L, Lopes M, Bitar A, Sichez JP, van Effenterre R: Medically intractable epilepsy from insular lowgrade gliomas: improvement after an extended lesionectomy. Acta Neurochir (Wien) 144:563-573, 2002

11. Englot DJ, Berger MS, Barbaro NM, Chang EF: Factors associated with seizure freedom in the surgical resection of glioneuronal tumors. Epilepsia 53:51-57, 2012

12. Englot DJ, Berger MS, Barbaro NM, Chang EF: Predictors of seizure freedom after resection of supratentorial low-grade gliomas. A review. J Neurosurg 115:240-244, 2011

13. Englot DJ, Berger MS, Chang EF, Garcia PA: Characteristics and treatment of seizures in patients with high-grade glioma: a review. Neurosurg Clin N Am 23:227-235, 2012

14. Englot DJ, Han SJ, Berger MS, Barbaro NM, Chang EF: Extent of surgical resection predicts seizure freedom in low-grade temporal lobe brain tumors. Neurosurgery 70:921-928, 2012

15. Englot DJ, Han SJ, Lawton MT, Chang EF: Predictors of seizure freedom in the surgical treatment of supratentorial cavernous malformations. Clinical article. J Neurosurg 115:1169-1174, 2011

16. Englot DJ, Wang DD, Rolston JD, Shih TT, Chang EF: Rates and predictors of long-term seizure freedom after frontal lobe epilepsy surgery: a systematic review and meta-analysis. Clinical article. J Neurosurg 116:1042-1048, 2012

17. Englot DJ, Young WL, Han SJ, McCulloch CE, Chang EF, Lawton MT: Seizure predictors and control after microsurgical resection of supratentorial arteriovenous malformations in 440 patients. Neurosurgery 71:572-580, 2012

18. Giulioni M, Galassi E, Zucchelli M, Volpi L: Seizure outcome of lesionectomy in glioneuronal tumors associated with epilepsy in children. J Neurosurg 102 (3 Suppl):288-293, 2005

19. Hoh BL, Chapman PH, Loeffler JS, Carter BS, Ogilvy CS: Results of multimodality treatment for 141 patients with brain arteriouvenous malformations and seizures: factors associated with seizure incidence and seizure outcomes. Neurosurgery 51:303-311, 2002

20. Hu WH, Ge M, Zhang K, Meng FG, Zhang JG: Seizure outcome with surgical management of epileptogenic ganglioglioma: a study of 55 patients. Acta Neurochir (Wien) 154:855861,2012

21. Janszky J, Pannek HW, Janszky I, Schulz R, Behne F, Hoppe
M, et al: Failed surgery for temporal lobe epilepsy: predictors of long-term seizure-free course. Epilepsy Res 64:35-44, 2005

22. Jehi LE, Irwin AI, Kayyali H, Vadera S, Bingaman W, Najm I: Levetiracetam may favorably affect seizure outcome after temporal lobectomy. Epilepsia 53:979-986, 2012

23. Khajavi K, Comair YG, Wyllie E, Palmer J, Morris HH, Hahn JF: Surgical management of pediatric tumor-associated epilepsy. J Child Neurol 14:15-25, 1999

24. Maschio M, Dinapoli L: Patients with brain tumor-related epilepsy. J Neurooncol 109:1-6, 2012

25. Moosa AN, Gupta A, Jehi L, Marashly A, Cosmo G, Lachhwani D, et al: Longitudinal seizure outcome and prognostic predictors after hemispherectomy in 170 children. Neurology 80:253-260, 2013

26. Morris HH, Estes ML, Gilmore R, Van Ness PC, Barnett GH, Turnbull J: Chronic intractable epilepsy as the only symptom of primary brain tumor. Epilepsia 34:1038-1043, 1993

27. Motamedi M, Nguyen DK, Zaatreh M, Singh SP, Westerveld $\mathrm{M}$, Thompson JL, et al: Levetiracetam efficacy in refractory partial-onset seizures, especially after failed epilepsy surgery. Epilepsia 44:211-214, 2003

28. Park YS, Kim DS, Shim KW, Kim JH, Choi JU: Factors contributing to resectability and seizure outcomes in 44 patients with ganglioglioma. Clin Neurol Neurosurg 110:667-673, 2008

29. Prayson RA: Diagnostic challenges in the evaluation of chronic epilepsy-related surgical neuropathology. Am J Surg Pathol 34:e1-e13, 2010

30. Rowland NC, Englot DJ, Cage TA, Sughrue ME, Barbaro NM, Chang EF: A meta-analysis of predictors of seizure freedom in the surgical management of focal cortical dysplasia. Clinical article. J Neurosurg 116:1035-1041, 2012

31. Rudà R, Trevisan E, Soffietti R: Epilepsy and brain tumors. Curr Opin Oncol 22:611-620, 2010

32. Schwartz TH, Spencer DD: Strategies for reoperation after comprehensive epilepsy surgery. J Neurosurg 95:615-623, 2001

33. Shamji MF, Fric-Shamji EC, Benoit BG: Brain tumors and epilepsy: pathophysiology of peritumoral changes. Neurosurg Rev 32:275-286, 2009

34. Southwell DG, Garcia PA, Berger MS, Barbaro NM, Chang EF: Long-term seizure control outcomes after resection of gangliogliomas. Neurosurgery 70:1406-1414, 2012

35. Vale FL, Pollock G, Benbadis SR: Failed epilepsy surgery for mesial temporal lobe sclerosis: a review of the pathophysiology. Neurosurg Focus 32(3):E9, 2012

36. von Lehe M, Wellmer J, Urbach H, Schramm J, Elger CE, Clusmann H: Insular lesionectomy for refractory epilepsy: management and outcome. Brain 132:1048-1056, 2009

37. You G, Huang L, Yang P, Zhang W, Yan W, Wang Y, et al: Clinical and molecular genetic factors affecting postoperative seizure control of 183 Chinese adult patients with low-grade gliomas. Eur J Neurol 19:298-306, 2012

38. You G, Sha ZY, Yan W, Zhang W, Wang YZ, Li SW, et al: Seizure characteristics and outcomes in 508 Chinese adult patients undergoing primary resection of low-grade gliomas: a clinicopathological study. Neuro Oncol 14:230-241, 2012

Manuscript submitted February 4, 2013.

Accepted March 8, 2013.

The case report featured in this paper is part of a poster submission to the 10th Biennial Satellite Tumor Symposium of the AANS, held in New Orleans, Louisiana, on April 26-27, 2013.

Please include this information when citing this paper: DOI: 10.3171/2013.3.FOCUS1348.

Address correspondence to: William C. Gump, M.D., Norton Neuroscience Institute, 210 East Gray Street, Suite 1102, Louisville, Kentucky 40202. email: william.gump@ nortonhealthcare.org. 